\title{
Cancer antigen prioritization: a road map to work in defining vaccines against specific targets. A point of view
}

\section{Daniel E. Gomez ${ }^{1 *}$, Ana María Vázquez ${ }^{2}$ and Daniel F. Alonso ${ }^{1}$}

\author{
1 Laboratory of Molecular Oncology, Quilmes National University, Buenos Aires, Argentina \\ 2 Center of Molecular Immunology, La Habana, Cuba \\ *Correspondence: degomez@unq.edu.ar
}

Edited by:

Amparo Macías, Center of Molecular Immunology, Cuba

The use of anti-idiotype antibodies as vaccines to stimulate antitumor immunity is a very promising pathway in the therapy of cancer. A good body of work in animal tumor models have demonstrated the efficacy of anti-Id vaccines in preventing tumor growth and curing mice with established tumors. A number of monoclonal anti-Id antibodies that mimic different human tumor-associated antigens (TAAs) have been developed and tested in the clinic, demonstrating interesting. In general terms, the antigen mimicry by anti-Id antibodies has reflected structural homology in the most of the cases, and amino acid sequence homology in a minority of them. The major challenge of immunotherapy using anti-idiotype vaccines is to identify the optimal anti-idiotype antibody that will function as a true surrogate antigen for a TAA system, and ideally will generate both humoral and cellular immune responses. Several clinical studies have shown enhanced patient's survival when receiving anti-Id vaccines, the true demonstration of efficacy of these vaccines will depend upon the results of several randomized Phase III clinical trials that are currently planned or ongoing (Bhattacharya-Chatterjee et al., 2002).

With the numerous antigens that can be used in immunotherapy the decision making process for researchers, hospitals, and companies, in whether or not invest resources in a specific antigen has been always a very complicated matter both for classic therapeutic vaccines and even more for anti-idiotype vaccines. Fortunately, in a recent work by the National Cancer Institute Translational Research Working Group (Cheever et al., 2009) was developed a method for prioritization of cancer antigens paving the way to take more rational, informed decisions. Such work aimed to develop a priority-ranked list of cancer vaccine target antigens based on predefined and preweigthed objective criteria. An additional aim was testing a new approach for prioritizing translational research opportunities based on an analytic hierarchy process (AHP), a structured technique and mathematical model for dealing with complex decisions. Antigen prioritization involved developing a list of "ideal" cancer antigen criteria/characteristics, assigning relative weights to those criteria using pairwise comparisons. The result of the criteria weighting, in descending order, was as follows: (a) therapeutic function, (b) immunogenicity, (c) role of the antigen in oncogenicity, (d) specificity, (e) expression level and percent of antigen-positive cells, ( $f$ ) stem cell expression, (g) number of patients with antigen-positive cancers, (h) number of antigen epitopes, and (i) cellular location of antigen expression.

Some authors have enunciated the introduction of potential biases in the National Cancer Institute Pilot Project (Lang et al., 2009). These authors affirmed that the methodology used (AHP), is not well described, and is subject to several sources of possible bias, such as participant selection, number of antigens chosen for prioritization, errors in rank order, redundancy, and internal validity. First of all, we differ with Lang et al. in the fact that AHP is not well described, beyond being a very well known technique properly used in a variety of settings, including cancer clinical decisions (Katsumura et al.,2008; see also Dolan and Iadarola, 2008). Cheever et al. clearly described the method by citing the popular work of Busham and Rai (2004) and how AHP is used in a Web-based tool (Olson et al., 2007). AHP is a powerful tool, used widely in science, and although has had some detractors over the years, Forman and Gass (2010) carried out an in-depth paper discussing and rebutting the academic criticisms of AHP.
Anti-idiotype cancer vaccines development is limited by several factors, including funding concerns. The work of Cheever et al. in our opinion can also be used as a map to develop stronger and faster evidence or in case of failure, deciding when to stop a project. The associated lists of weighted criteria enumerated in Cheever's paper inform investigators as to what experimental evidence is required to advance antigens to higher priority levels, therefore, improving its chance to pass to translational research. Also, the lack of superb data, especially in the therapeutic function, could be multifactorial, including inadequate trial design or patient selection and inadequate vaccine formulation or regimens. These deficiencies can be overcome by more intelligent trial design based on assessment of past "productive failures."

Having that work as a reference, we analyzed the data available for the anti-idiotype vaccine Racotumomab (formerly known as 1E10) and its target antigen, $N$-glycolyl (NGc)-containing gangliosides and particularly NGcGM3 (reviewed in detail by Fernandez et al., 2010). As shown in Table 1, the antigen match all criteria considered at least in some proportion, having a cumulative score of 0.62 . Interestingly, with this score NGcGM3 ranked within the top 15th cancer antigens selected by Cheever et al., with a score similar to the GD2 ganglioside, the Melan A/MART1 gene product, or the carcinoembrionic antigen.

At the time of Cheever's publication no cancer vaccine was yet approved by FDA. However, recent approval of sipeleucel-T for men with advanced prostate cancer targeting PAP antigen, gave us a valuable lesson on this matter (Bot, 2010). Interestingly, PAP ranked 26 out of 75 antigens in the ranking of cancer antigen pilot prioritization, confirming its capacity to somehow 
Table 1 | Relevant characteristics and score for NGcGM3 ganglioside as a cancer antigen, according to the antigen prioritization criteria described by Cheever et al. (2009).

\begin{tabular}{|c|c|c|}
\hline Criteria & Subcriteria for NGcGM3 & $\begin{array}{l}\text { Score (total weight } \\
\text { of criteria) }\end{array}$ \\
\hline Therapeutic function & Adequate data, controlled vaccine trial suggestive & $0.27(0.32)$ \\
\hline Immunogenicity & T-cell and antibody responses elicited in clinical trials & $0.17(0.17)$ \\
\hline Oncogenicity & $\begin{array}{l}\text { Increased expression correlated with survival and advanced disease, but oncogenic } \\
\text { function need to be clarified }\end{array}$ & $0.03(0.15)$ \\
\hline Specificity & Overexpressed in cancer with little or no expression in normal adult tissues & $0.05(0.15)$ \\
\hline Expression level and\% positive cells & Highly expressed on most cancer cells in patients designated for treatment & $0.02(0.07)$ \\
\hline Stem cell expression & Expression on most cancer cells but without information about putative stem cells & $0.01(0.05)$ \\
\hline No. patients with antigen-positive cancers & High level of expression in many patients with a particular tumor type & $0.04(0.04)$ \\
\hline No. antigen epitopes & Short antigenic segment with one or few epitopes & $0.01(0.04)$ \\
\hline Cellular location of antigen expression & Expressed on the cell surface with little or no circulating antigen & $0.02(0.02)$ \\
\hline Cumulative score for NGcGM3 & & $0.62(1.00)$ \\
\hline
\end{tabular}

"forecast" those antigens more likely to be translated to patients. Although the ranking is dynamic, given that priorities change as knowledge accrues from new studies, we must reinforce the idea that the associated lists of weighted criteria inform investigators as to what experimental evidence is required to advance antigens to higher priority levels, and even more, if the antigen of choice is relevant. Always perfectible, those criteria helped us to evaluate that NGcGM3 comprised most if not all the criteria. Therefore NGc-containing gangliosides are antigens worth investing in the acceleration of its translational research.

\section{ACKNOWLEDGMENTS}

Daniel E. Gomez and Daniel F. Alonso are members of the National Research Council (CONICET, Argentina). Dr. Daniel E. Gomez belongs to the National Cancer Institute of Argentina.

\section{REFERENCES}

Bhattacharya-Chatterjee, M., Chatterjee, S. K., and Foon, K. A. (2002). Anti-idiotype antibody vaccine therapy for cancer. Expert Opin. Biol. Ther. 8, 869-881.

Bot, A. (2010). The landmark approval of Provenge, what it means to immunology and "in this issue": the complex relation between vaccines and autoimmunity. Int. Rev. Immunol. 29, 235-238.

Busham, N., and Rai, K. (2004). Strategic Decision Making: Applying the Analytic Hierarchy Process. London: Springer-Verlag.

Cheever, M. A., Allison, J. P., Ferris, A. S., Finn, O. J., Hastings, B. M., Hecht, T. T., Mellman, I., Prindiville, S. A., Viner, J. L., Weiner, L. M., and Matrisian, L. M. (2009). The prioritization of cancer antigens: a National Cancer Institute pilot project for the acceleration of translational research. Clin. Cancer Res. 15, 5323-5337.

Dolan, J. G., and Iadarola, S. (2008). Risk communication formats for low probability events: an exploratory study of patients preferences. BMCMed. Inform. Decis. Mak. 8, 14-23.

Fernandez, L. E., Gabri, M. R., Guthmann, M.D., Gomez, R.E., Gold, S., Fainboim, L., Gomez, D. E., and Alonso, D. F. (2010). NGcGM3 ganglioside: a privileged target for cancer vaccines. Clin. Dev. Immunol. 2010, 814397. Forman, E. H., and Gass, S. I. (2010). The analytical hierarchy process-an exposition. Oper. Res. 49, 235-238.
Katsumura, Y., Yasunaga, H., Imamura, T., Obe, K., and Oyama, H. (2008). Relationship between risk information on total colonoscopy and patient preferences for colorectal cancer screening options: analysis using the analytic hierarchy process. BMC Health Serv. Res. 21, 106-114.

Lang, J. M., Andrei, A. C., and Mc Nee, D. G. (2009). Prioritization of cancer antigens: keeping the target in sight. Expert Rev. Vaccines 8, 1657-1661.

Olson, W.C., Heston, W. D., and Rajasejaran, A. K. (2007). Clinical trials of cancer therapies targeting prostatespecific membrane antigens. Rev. Recent Clin. Trials 2,182-190.

Received: 31 May 2012; accepted: 11 June 2012; published online: 28 June 2012.

Citation: Gomez DE, Vázquez AM and Alonso DF (2012) Cancer antigen prioritization: a road map to work in defining vaccines against specific targets. A point of view. Front. Oncol. 2:66. doi:10.3389/fonc.2012.00066

This article was submitted to Frontiers in Tumor Immunity, a specialty of Frontiers in Oncology.

Copyright (c) 2012 Gomez, Vázquez and Alonso. This is an open-access article distributed under the terms of the Creative Commons Attribution Non Commercial License, which permits non-commercial use, distribution, and reproduction in other forums, provided the original authors and source are credited. 